\title{
Eye blink elicitation and measurement in the human infant
}

\author{
ROGER R. MARSH \\ Children's Hospital of Philadelphia, Philadelphia, Pennsylvania 19131 \\ HOWARD S. HOFFMAN \\ Bryn Mawr College, Bryn Mawr, Pennsylvania 19010 \\ and \\ CHRISTOPHER L. STITT \\ Department of Industrial Relations and Organizational Behavior, \\ Temple University, Philadelphia, Pennsylvania 19122
}

\begin{abstract}
Eye blinks elicited by a mechanically produced tap to the glabella (the flattened area between the eyebrows) are monitored by a device that projects low-intensity infrared light, which is reflected from the eyelid and detected by a phototransistor. Appropriate filtering and decoding circuitry insures reliable tracking of responses. Comparison of the optical eye blink monitor with a minitorque potentiometer monitor showed the two devices to yield similar recordings.
\end{abstract}

The eye blink is readily elicited by a variety of stimuli, as well as by instruction, and it may be measured reliably with any one of a number of transducers. In the testing of infants and young children, however, the traditional stimulation and measurement devices have proven awkward and perhaps even hazardous. We have been particularly concerned with the problems involved in linking a transducer to the eyelid and with the difficulty of presenting a stimulus sufficiently intense to reliably elicit an eye blink, yet not so aversive as to try the volatile temper of a baby. Our solutions to these problems have proven most satisfactory with respect to our requirements of safety, comfort, and reliability.

Eye blinks are elicited by a brisk tap to the glabella, the region between the eyebrows. This stimulus can elicit a vigorous blink even in infants who prove unresponsive to periocular air puff. A small optical monitor is used to assess blinks. The monitor eliminates the requirement of mechanical attachment to the eyelid, with attendant risk of trauma, irritation, or infection.

\section{GLABELLA STIMULATOR}

The blink-eliciting device (the tap unit) consists of a small light-weight solenoid mounted on the forehead by means of a flexible headpiece that also carries the

The development of these devices occurred during the course of a research project supported by NIH Grant HD 10511. We wish to thank Alan Taren for his early contribution to the design of the circuits for the optical detector. We also wish to thank Coulbourn Instruments, Inc., for suggesting modifications to improve those circuits. Requests for reprints should be sent to Howard S. Hoffman, Department of Psychology, Bryn Mawr College, Bryn Mawr, Pennsylvania 19010. optical monitor. Figures 1,2 , and 3 show the details of the tap unit (Figure 1), the optical monitor (Figure 3), and the flexible headpiece to which they are fastened (Figure 2). The tap unit (Figure 1) is constructed from the $12-\mathrm{V}$ solenoid used to operate a remote switch in an HO-guage model train layout. The device is manufactured by Atlas Tool Company, Inc., Hillside, New Jersey, and is available in most hobby shops at a cost of approximately $\$ 2$. Construction of the tap unit requires that the switch be disassembled and that one of the two windings on the solenoid's coil be discarded. The other winding is connected to a pair of stranded 32-guage "earphone" wires that exit from the solenoid through a $3-\mathrm{cm}$ length of brass tubing that is fastened to the solenoid by a generous application of epoxy cement. The slug of the solenoid is extended by a small length of wooden dowel that terminates in a ball of silastic rubber (see the right side of Figure 1). To insure its prompt

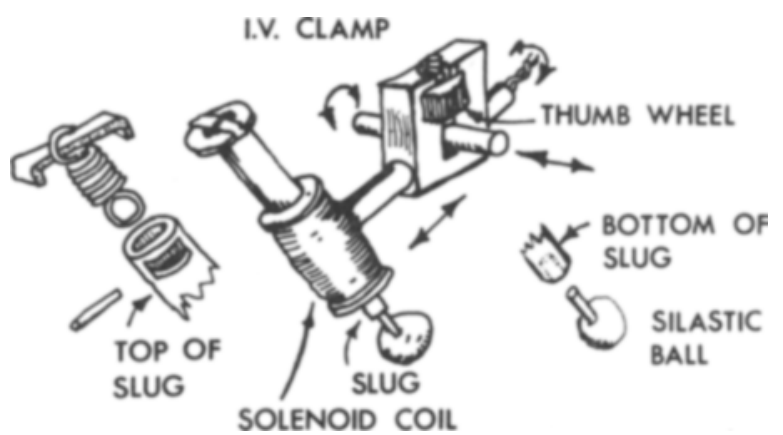

Figure 1. Tap unit. A small solenoid with a silicone nubber ball mounted on its slug delivers a tap to the glabella when the coil is energized briefly. The spring at the top of the slug returns it after a tap has been delivered. 

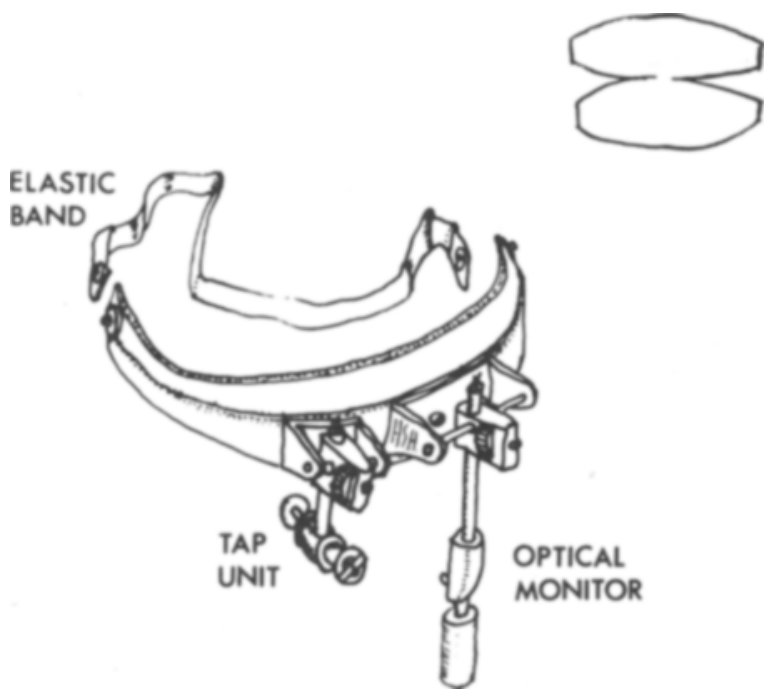

Figure 2. Headpiece. This unit is constructed of expanded polyvinyl chloride cut in the pattern shown at the upper right.

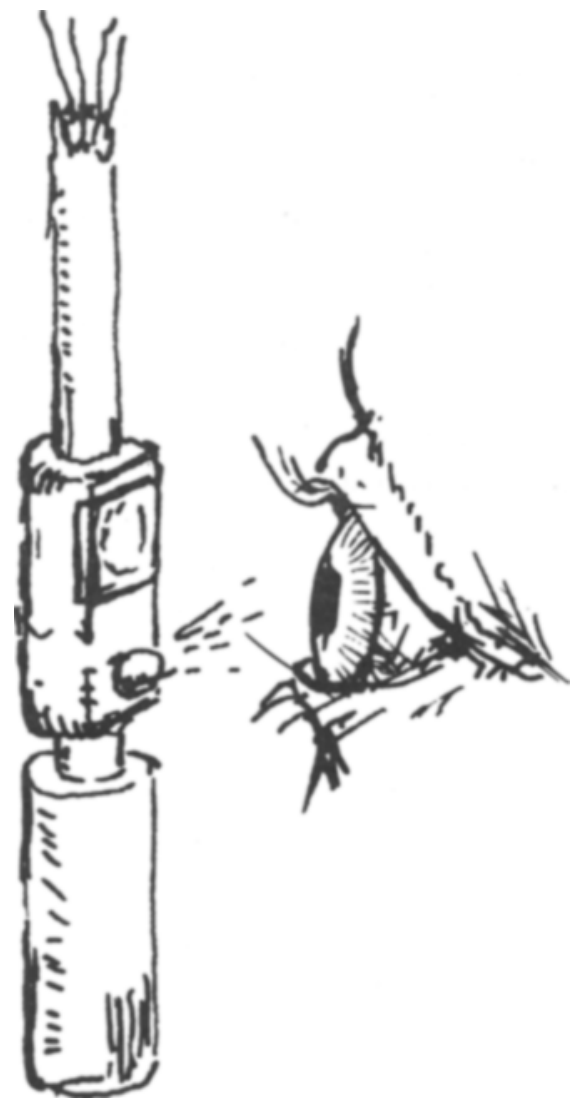

Figure 3. Optical monitor. The light-emitting diode (bottom) projects a weak infrared beam to the eye. The phototransistor (covered by a small square of Wratten infrared filter) detects inf rared light reflected from the eyelid when it covers the eye.

return after a given tap, the upper end of the slug is permanently fastened to a light spring that is fixed to the end of the solenoid, as shown on the left side of Figure 1. When the coil of the solenoid is energized, the slug moves forward to its limit (halfway out of the coil) at a rate that is proportional to the applied voltage. In practice, a brief pulse $(50 \mathrm{msec})$ of $10-15 \mathrm{~V}$ dc is adequate to reliably elicit a blink in most infants.

The headpiece (Figure 2) is constructed of expanded polyvinyl chloride of the sort used to make table mats. It is cut in the pattern shown and glued together in an inverted-V configuration. This configuration provides good conformation to various sizes and shapes of heads, and it supplies a rigid enough substrate to provide good mechanical isolation between the optical monitor and the tap unit.

Figure 2 shows the manner in which the tap unit and the optical monitor are attached to the headpiece. Each unit is supported by a small piece of sheet aluminum that is bent into a U-shaped configuration. A solid metal rod covered with a length of plastic tubing is securely fastened across the arms of each aluminum support. The brass tubes that extend from the tap unit and from the optical monitor are held against these rods by small plastic clamps of the sort used to control the flow in an intravenous (IV) feeding system. As illustrated in Figure 1, the advantage of this arrangement is that each unit can move in all directions when the thumbwheel of its IV clamp is loose, and yet the unit is completely rigid once that thumbwheel is tightened.

To date, we have constructed and tested a half dozen headpieces with associated tap unit and optical monitor. We have found them to be entirely acceptable to subjects ranging in age from 4 weeks to 77 years. The units are easy to put on and take off, and unless actively dislodged (by brushing against another object), they stay in adjustment.

\section{RESPONSE TRANSDUCER}

Optical devices for measuring eye blinks have typically employed a remote light source. Since the measured signal depends jointly on incident light and on the reflectance between cornea and lid, other investigators have employed a bridge circuit in which one photocell measures reflected light at the orbit and another, a reference source, measures reflected light from a nearby patch of skin. The ratio between the signals then indicates the difference in reflectance between the two target areas, independent of ambient illumination (DeLucia, 1968).

We elected a different approach to this problem. The light source, an infrared light-emitting diode (LED), is incorporated in the transducer assembly so that illuminance is independent of head position. In order to render the device insensitive to ambient light, the output of the LED is modulated with a $5-\mathrm{kHz}$ signal. The output of the phototransistor is in turn filtered so that low-frequency components (e.g., $60 \mathrm{~Hz}$ and its harmonics) are rejected, and only the $5-\mathrm{kHz}$ component of the reflected signal is detected.

\section{The Optical Monitor}

The light source and detector are mounted in a small 
block of Plexiglas, as shown in Figure 3. The source, an inf rared LED (Type TIL32) with peak output at $940 \mathrm{~nm}$, is the lower of the two devices. Its optical axis is tilted $15 \mathrm{deg}$ above horizontal. The reflected signal is detected by a phototransistor (Type FPT-100) that is recessed and completely covered with an infrared filter that passes all wavelengths greater than $850 \mathrm{~nm}$ (Kodak Wratten gelatin Filter $87 \mathrm{C}$ ). The device is suspended from the previously described headpiece by the rigid brass tube that extends upward in Figure 3. This tube carries the wiring for the assembly. Another segment of brass tubing, sheathed in latex tubing, projects $2.5 \mathrm{~cm}$ below the assembly. This extension insures that the monitor cannot enter the orbit.

\section{Circuitry}

The electrical circuits for the optical monitor consist of a current source modulated by a $5-\mathrm{kHz}$ signal that drives the LED and an amplifier-filter-demodulator circuit that processes the signal from the phototransistor. Both units are powered by a 12-V supply.

Transmitter circuit. This device (shown in Figure 4)

\section{TRANSMITTER}

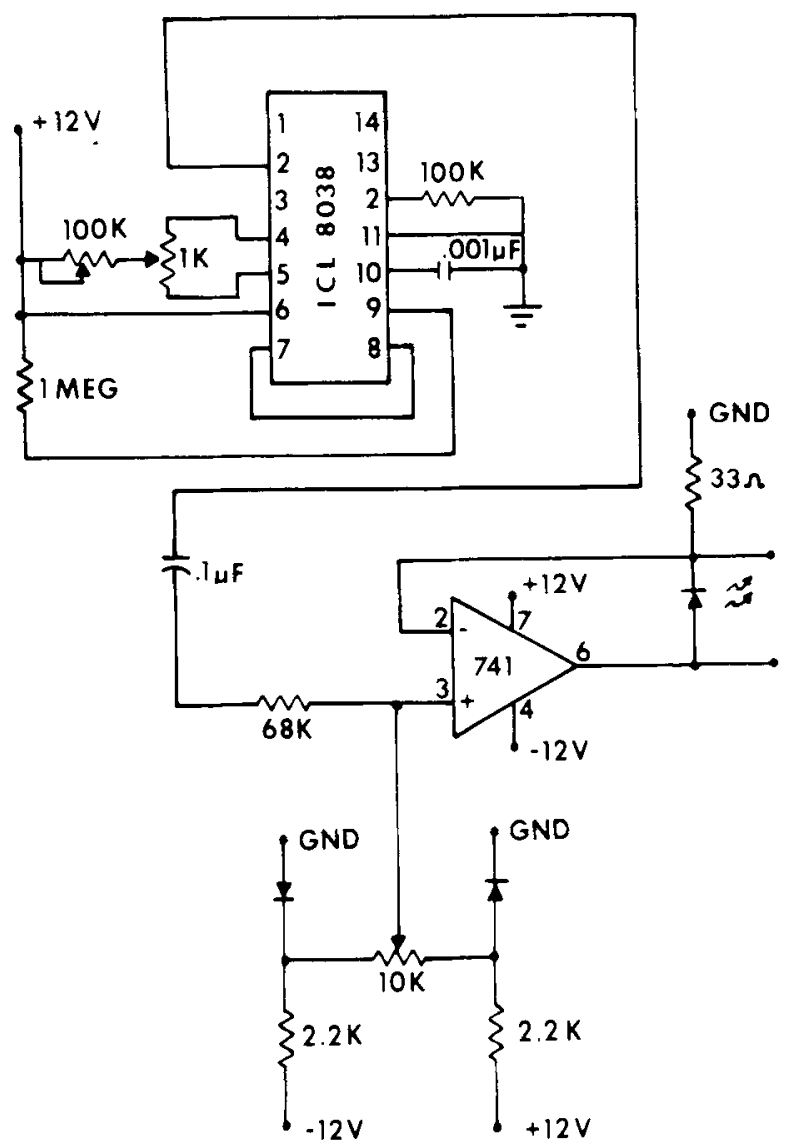

Figure 4. Transmitter circuit. This circuit drives the lightemitting diode. It employs a solid state function generator that is adjusted to produce a $5-\mathrm{kHz}$ sine wave.
DETECTOR
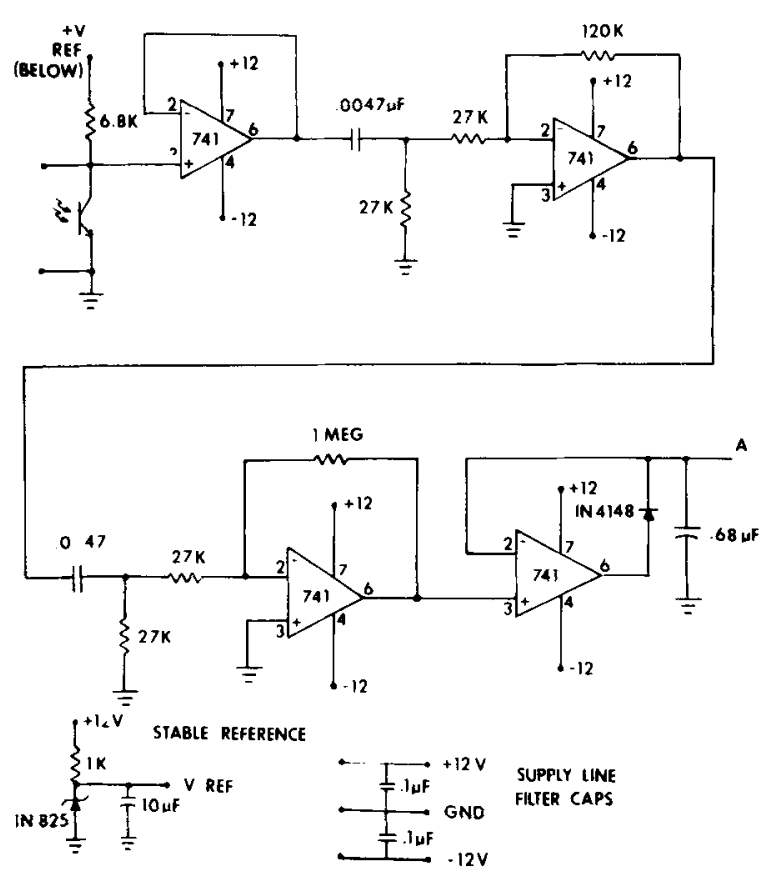

Figure 5. The initial segment of the detector circuit. This section of the circuit consists of a voltage follower, a two-stage filter and amplifier that rejects signals with frequencies below $5 \mathrm{kHz}$ and amplifies $5-\mathrm{kHz}$ signals, a half-wave rectifier circuit, and a gain-of-two amplifier.

employs an ICL 8036 function generator that is adjusted to produce a $5-\mathrm{kHz}$ sine wave. Frequency adjustment is made with the 100 -kohm variable resistor, and symmetry of the waveform, by the $1-\mathrm{kohm}$ variable resistor. The 10 -kohm variable resistor determines the $\mathrm{dc}$ offset of the output from an operational amplifier (Type 741).

Detector circuit. The detector circuit (see Figures 5 and 6) employs the phototransistor and six IC operational amplifiers (all Type 741). Operation is as follows: The first IC is a voltage follower measuring the potential across the phototransistor of the detector circuit. The use of a separate Zener-controlled reference voltage renders the input signal largely insensitive to power supply variations. The second and third ICs, and the $\mathrm{RC}$ sections on their inputs, form a two-stage high-pass filter and amplifier. Low-frequency signals from ambient light sources are rejected, and the $5-\mathrm{kHz}$ component is amplified approximately 165 times. The next stage, a half-wave rectifier circuit with a capacitor at the output, demodulates the signal. The fifth IC simply serves as a constant-impedence gain-of-two amplifier. This is followed by a high-pass filter, to stabilize the baseline, and a final variable-gain amplifier.

The detector circuit is somewhat sensitive to transients, such as those associated with the operation of the glabella stimulator. The usual precautions-adequate shielding, avoidance of ground loops, and minimal input lead length-are recommended. 


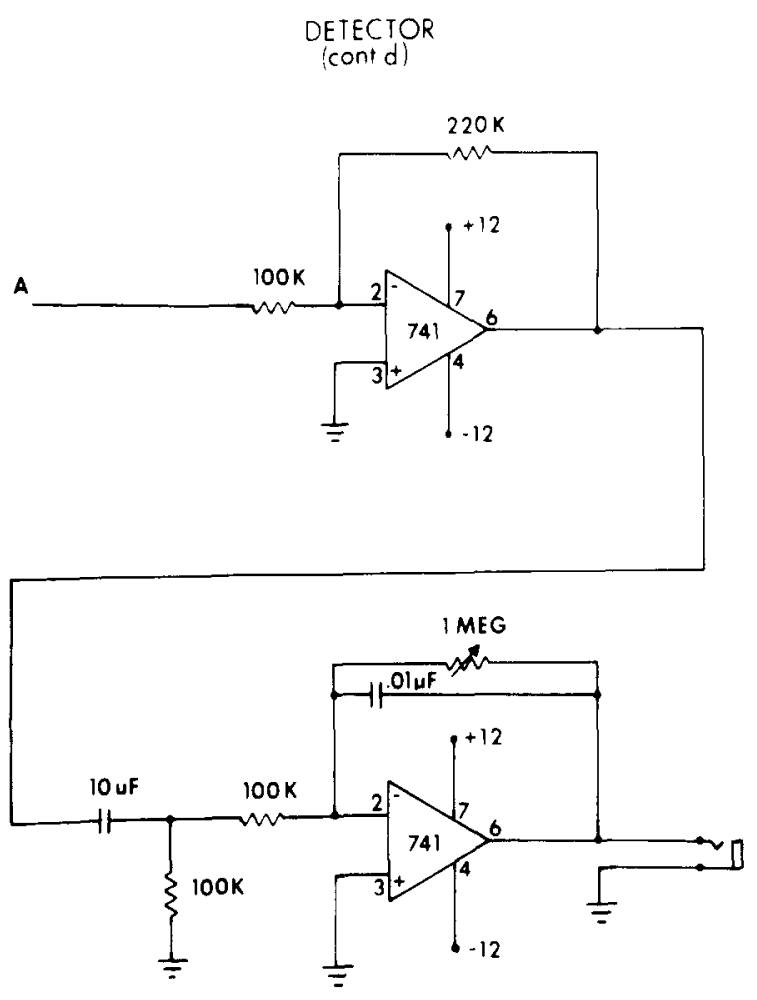

Figure 6. The final segment of the detector circuit. The first of these units stabilizes the baseline, and the last consists of a final variable-gain amplifier.

\section{PERFORMANCE}

Some practice is required to learn the correct placement of the optical monitor, but when properly positioned, its sensitivity compares well with that of the lowtorque potentiometer (the device most commonly used to measure eye blinks). Figure 7 shows typical instructed eye blinks measured simultaneously by a minitorque potentiometer linked to the right eyelid and the optical device positioned about $1 \mathrm{~cm}$ in front of the left eye. Figure 8 demonstrates the linearity of the device for the same subject, again with simultaneous measurement of instructed blinks.

The frequency response of the transducer was characterized by placing an eccentric white disk on a color wheel and positioning the detector to measure cyclic variations in reflectance as the wheel rotated. Frequency response was flat, within $1 \mathrm{~dB}$ from .5 to $16 \mathrm{~Hz}$. Variation in the level of ambient illumination during this procedure had little effect. Using either incandescent or fluorescent lights to produce illumination levels ranging from 0 to $50 \mathrm{fc}$ affected the response by less than $10 \%$. (In practice, a slightly greater effect of ambient illumination may be encountered, owing to variations in reflectance of the iris as the pupil dilates or contracts.) No $60-$ or $120-\mathrm{Hz}$ noise was measurable with either light source.

\section{SAFETY}

A major incentive in developing this system was concern over possible infection or trauma with the stimulating and recording procedures traditionally employed in such work. The corneal or periorbital air puff may propel contaminants into the baby's eye, and the fitting of mechanical linkages to the eyelid provides opportunity for cross-infection and irritation of sensitive tissues. Conjunctivitis is, in our opinion, a real and underestimated problem in pediatric research, which so often is conducted in hospitals and outpatient facilities. It is clear from the above description that the design of the present system eliminates these safety problems.

The greatest safety concerns with the present device and any system employing near infrared radiation (700-1400 nm) are possible effects upon the lens of the eye (cataractogenesis) and the possibility of retinal thermal injury. The Occupational Safety and Health Administration (OSHA) has established radiation limit

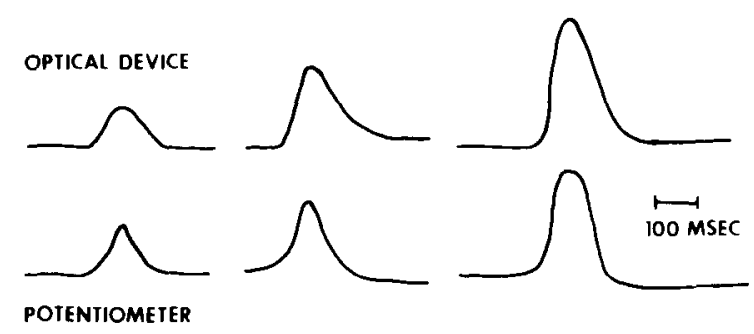

Figure 7. Tracings of simultaneous oscilloscope recordings from the optical monitor (on the left eye) and a minitorque potentiometer (on the right eye) during instructed blinks.

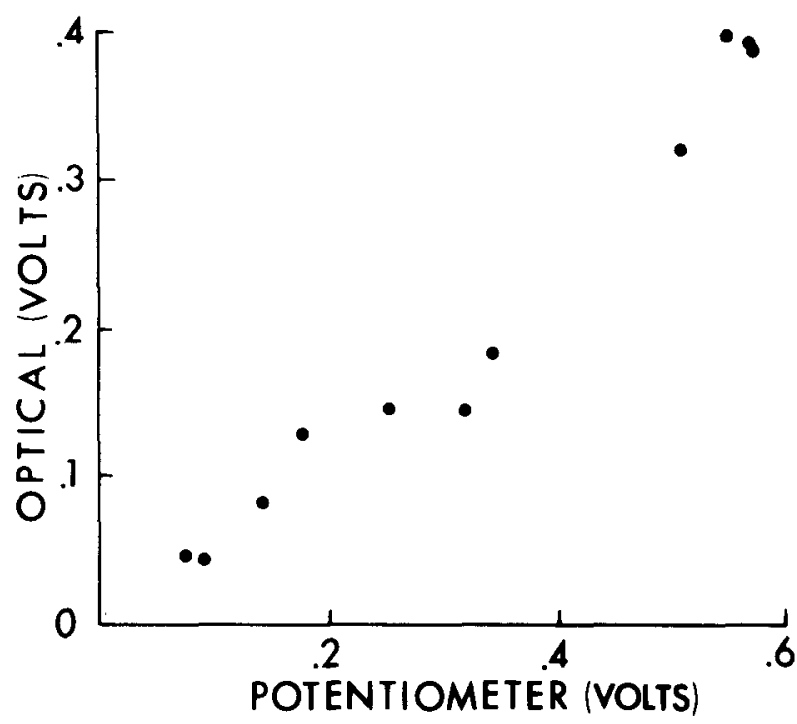

Figure 8. Scatterplot showing the peaks of a series of simultaneous recordings obtained from the optical monitor (left eye) and a minitorque potentiometer (right eye) when a subject was instructed to produce blinks of various magnitudes. 
values that represent conditions to which it is believed individuals may be exposed without adverse effects of either type. These standards are for distant or collimated sources where an important danger is that the image of the source might be focused on the retina. Because the infrared source (the LED) device described here is located fairly close to the eye (approximately $1 \mathrm{~cm}$ ), its image cannot be brought into focus; instead, it floods the retina with a diffuse beam. According to the manufacturer's specifications, the LED has a total radiant output of $1.2 \mathrm{~mW}$. The peak of this output is at $940 \mathrm{~nm}$, and the half-power points are approximately $60 \mathrm{~nm}$ above and below this value. If one assumes that the entire output of the device falls on the lens of the eye, the radiant flux striking the lens or the retina cannot exceed $1.2 \mathrm{~mW} / \mathrm{cm}^{2}\left(.29 \mathrm{mcal} / \mathrm{sec} / \mathrm{cm}^{2}\right)$. This value is well below OSHA's figure for safe levels of near infrared radiation on the lens $\left(10 \mathrm{~mW} / \mathrm{cm}^{2}\right)$. In regard to thermal injury, a retinal exposure of $1.2 \mathrm{~mW} / \mathrm{cm}^{2}$ of $940-\mathrm{nm}$ light is the equivalent of $.06 \mathrm{~cd} / \mathrm{cm}^{2}$ of white light. According to OSHA recommendations, white light of less than $1 \mathrm{~cd} / \mathrm{cm}^{2}$ may be regarded as safe, and the hazard of infrared light is less by an order of magnitude.

Finally, an empirical demonstration of the safety of the device can be made by measuring the output of the voltage-follower amplifier (in the detector circuit) when the phototransistor is exposed to various sources of light. This can be done both with and without the Wratten filter in place. Since the bandpass characteristics of the filter closely approximate the energy band of the LED, measures taken with the filter in place provide an index of only the near infrared provided by a given source, whereas measures taken without the filter provide an index of the source's radiation across the larger band that includes much of the visible spectrum.

Using the LED as the source yields a maximum reading when the LED is physically touching the phototransistor, and it drops appreciably when the LED is at a distance of $1 \mathrm{~cm}$. At any distance, however, this reading is unaffected by insertion or withdrawal of the filter. This observation is consistent with the fact that the bandwidth of the filter closely approximates the bandwidth of the source. It is also consistent with the observation that, using a $60-\mathrm{W}$ incandescent lamp as the source, insertion and removal of the filter makes a noticeable difference; higher readings are obtained when the filter is removed.

When the LED is in physical contact with the phototransistor, the output of the phototransistor is approximately the same as when the phototransistor reads the light reflected from a sheet of white paper that is illuminated by a 60-W incandescent bulb at a distance of $45 \mathrm{~cm}$; it is smaller than the output obtained when the filter is removed. This implies that the optical monitor offers no greater hazard from near infrared radiation than is encountered when reading. When radiation in the visible spectrum is included, it offers less hazard.

\section{REFERENCE}

DeLucia, C. A. Apparatus for recording eyeblink. Journal of Experimental Child Psychology, 1978, 6, 427-430.

(Accepted for publication July 26, 1979.) 